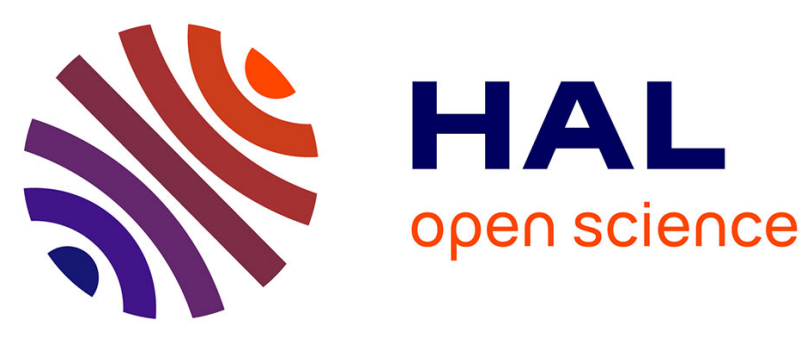

\title{
La Valeur dans les Lettres
}

Dominique Vaugeois

\section{To cite this version:}

| Dominique Vaugeois. La Valeur dans les Lettres. Modernités, 2007. hal-01999845

\section{HAL Id: hal-01999845 \\ https://hal.science/hal-01999845}

Submitted on 30 Jan 2019

HAL is a multi-disciplinary open access archive for the deposit and dissemination of scientific research documents, whether they are published or not. The documents may come from teaching and research institutions in France or abroad, or from public or private research centers.
L'archive ouverte pluridisciplinaire HAL, est destinée au dépôt et à la diffusion de documents scientifiques de niveau recherche, publiés ou non, émanant des établissements d'enseignement et de recherche français ou étrangers, des laboratoires publics ou privés. 


\title{
La Valeur dans les lettres
}

\author{
Dominique Vaugeois
}

Publié dans : L’Art et la question de la valeur, Modernités, dir. D. Rabaté, n² 25, 2007, p. 27-39.

En France, le débat sur la valeur s'est concentré, dans les dix dernières années, autour de la production artistique contemporaine (arts plastiques et littérature), c'est en somme sa face la plus visible, sa face publique autour par exemple de l'affaire Jourde et des nombreuses prises de position de Jean-Marie Domecq. Cependant, de façon moins ostensible mais non moins radicale, la question de la valeur sous la forme d'une interrogation sur le jugement de valeur a divisé en profondeur, durant la dernière décennie du $\mathrm{XX}^{\mathrm{e}}$ siècle, la réflexion esthétique au sens large ${ }^{1}$, c'est-à-dire aussi bien les philosophes de la discipline que les littéraires ${ }^{2}$ entre lesquels le polyvalent Gérard Genette fait le lien. D'autre part, sous l'influence des « cultural studies » et de la sociologie de l'art post-bourdieusienne, notamment celle que propose Nathalie Heinich, la question de la valeur (comme catégorie philosophique de la pensée et non comme simple qualité d'une œuvre) s'est progressivement imposée après des années d'oubli dans le domaine des études littéraires. C'est ainsi par exemple qu'une des tendances majeures en histoire littéraire s'inscrit dans le paradigme de la valeur, que ce soit pour réévaluer le canon (revoir l'histoire à l'envers, du point de vue de la figure de l'antimoderne, « sel du moderne $^{3} \gg$ ) ou répondre à l'interrogation contemporaine sur la valeur de la littérature elle-même ${ }^{4}$. Le point de vue de la valeur (au singulier ou au pluriel), dans ses implications à la fois culturelles, philosophiques, politiques et sociologiques, est désormais le garde-fou privilégié de l'universitaire vigilant : le champ définitionnel et d'application de la valeur, ses usages et ses fonctions, les modalités de

1. Voir par exemple Convergences et divergences esthétiques publié sous la direction de Danielle Cohen-Lévinas en 2001

2. Jean-Jacques Lecercle et Ronald Shusterman L'Emprise des signes. Débat sur l'expérience littéraire, Le Seuil « Poétique » 2002.

3. Antoine Compagnon, Les Antimodernes : de Joseph de Maistre à Roland Barthes, Gallimard, 2005, Collection « Bibliothèque des Idées », p. 448.

4. William Marx, L'Adieu à la littérature. Histoire d'une dévalorisation. XVIIIe-XXe siècle, Les Editions de Minuit, 2005, Collection « Paradoxe». 
sa production, ses « supports », ces divers niveaux d'analyses, souvent difficiles à distinguer, irriguent d'une manière ou d'une autre, directement ou indirectement voire par défaut, la réflexion contemporaine sur la littérature. Or il semble que la place de la valeur et de l'évaluation en littérature n'aille pas de soi. Invoquer la valeur en littérature, c'est faire apparaître au choix le spectre du conversatisme réactionnaire, celui d'une « sociologisation de la littérature » ou bien celui de l'approximation impressionniste. Ce que nous voudrions examiner ici, c'est à la fois ce que les pratiques universitaires et disciplinaires font à la valeur et inversement ce que la question de la valeur fait à la discipline littéraire, à son enseignement et à sa théorisation, ce qu'elle provoque mais aussi ce qu'elle met au jour d'impensé.

\section{Relativisme théorique et essentialisme pratique}

La pensée de la valeur aujourd'hui, telle qu'elle est réapparue à l'horizon des lettres dans le sillage des cultural studies est une pensée anti-essentialiste qui participe d'un modèle économique dont Jean-Joseph Goux ${ }^{5}$ a parfaitement montré le développement : à partir de 1870 et des théories des économistes néo-classiques qui accompagnent la naissance du second capitalisme, celui non plus de l'industrie mais de la banque, la pensée de la valeur dans le domaine de l'éthique, de l'esthétique, de la sémiotique, est tributaire d'un modèle boursier qui remplace définitivement (car nous ne sommes pas sortis de ce paradigme que « radicalise » mais ne crée pas ce qu'on appelé la pensée post-moderne de la fin des années 1960, Derrida, Baudrillard) l'ancienne philosophie, directement héritée de la métaphysique de Platon, de la valeur comme repère universel, invariable dont la fonction de mesure fixe les valeurs particulières. Dans le modèle boursier, la valeur est nécessairement, rappelle Goux, valeur mobilière et valeur d'échange, c'est-à-dire déterminée par un équilibre momentané qui fluctue selon les lois de l'offre et de la demande. Valéry constatait déjà non sans amertume que « la littérature est en proie à une perpétuelle activité toute semblable à celle de la Bourse. Il n'y est question que de valeurs, que l'on on introduit, que l'on exalte, que l'on rabaisse, comme si elles fussent comparables entre elles, ainsi que le sont en bourse les industries et les affaires les plus différentes du monde, une fois substitués par des signes ${ }^{6}$. » Or ce n'est pas la même chose d'admettre qu'économie et littérature sont liées, que la valeur littéraire est influencée par la réalité économique, seconde phase de la sociologie de l'art, bien assimilée, elle, par les littéraires, et d'admettre que la valeur littéraire est une valeur économique comme une autre dont le modèle ultime est monétaire. Il y a en effet une morale de l'art tenace qui l'oppose au mercantilisme, une idée de la communication littéraire, qu'elle se fasse de l'auteur au lecteur ou

5. Frivolité de la valeur, Essai sur l'imaginaire du capitalisme, Blusson, 2000.

6. Cité par Jacques Charpier, Paul Valéry (1956), Seghers, 1970, p. 140-141. 
du professeur à l'élève, qui se définit contre le modèle de consommation. C'est ce qui explique d'ailleurs la résistance que les littéraires ont longtemps manifestée aux théories de Bourdieu et de son école, en raison de ce cynisme - le refus du cynisme c'est bien le refus de la disparition des hiérarchies - qui assimile le littéraire à des stratégies de conquête et de domination. La littérature résiste plus longtemps que les arts plastiques à ce que la sociologie veut lui infliger, car le domaine des arts plastiques est de façon beaucoup plus évidente, ou moins contestable, exposé à la loi du marché.

L'ouvrage de référence de Barbara Herrnstein Smith, Contingencies of value ${ }^{7}$ publié en 1988, reprenant les schémas économiques, va pousser jusqu'à ses plus extrêmes conséquences le point de vue relativiste dont nous sommes pourtant depuis longtemps déjà familiers afin de nous en faire prendre la pleine mesure. En effet, le problème n'est plus tellement que la valeur est relative à celui qui prononce le jugement (c'est la vieille question de la subjectivité, celle de Hume et de Kant et l'antienne : des goûts et des couleurs...), ce qui lui garderait encore une certaine « authenticité » ou que la valeur de la littérature est différente selon les époques - ce que l'on admet sans difficulté -, mais qu'elle est relative aux circonstances dans lesquelles s'énoncent le jugement et qui sont de trois sortes : le cours des valeurs du moment, le but du jugement de valeur, et les destinateurs considérés. Or, si nous avons pris acte de la relativité du canon et si l'approche de la littérature à laquelle nous souscrivons est soucieuse d'éviter tout jugement normatif, néanmoins, la dynamique de la transmission qui fonde l'enseignement suppose un discours de légitimation. Si le modèle de la vente paraît excessif, il est certain que face à une audience pour qui de moins en moins la valeur de La Vie de Marianne s'impose comme une évidence, ou faudrait-il dire pour qui la valeur de La Vie de Marianne dépend presque exclusivement de sa place dans le cursus et de la parole de l'enseignant, il s'agit bien de justifier un choix, de rendre valable l'objet afin que l'échange puisse avoir lieu. Jean-Marie Schaeffer place le domaine de la valeur dans celui des échanges sociaux et nos pratiques universitaires s'inscrivent bien dans cette sphère. Or nos discours, examinés à la lumière de la pensée des valeurs, ne peuvent qu' apparaître hautement suspects : d'un côté nos pratiques relèvent inévitablement de logiques de savoir sur lesquelles désormais le soupçon a été porté, et, de l'autre côté, la prise de distance critique vis-à-vis de ces logiques de savoir au nom d'une conscience renouvelée du jeu des valeurs nous place sans ménagements devant la réalité des logiques de pouvoir, celle de l'empire moral que nous exerçons et qui nous semble alors relever du même inévitable dogmatisme tout aussi incompatible avec notre idée de l'enseignement. J'en prendrai trois exemples (il y en a d'autres que met en lumière l'argumentation de Barabara

7. Barbara Herrnstein Smith, Contingencies of value. Alterrnative Perspectives for Critical Theory. Harvard University Press, 1988 
Herrnstein Smith), parce que chacun engage plusieurs versions de la valeur : les valeurs qu'une société partage et qui sont impliquées dans l'expérience littéraire, la valeur - littéraire ou non - du texte, la valeur de la littérature elle-même.

Tout d'abord, le discours que nous tenons sur les textes soumis à l'analyse est suspendu dans la plupart de ses manifestations à l'idée de la richesse ou bien de la complexité du texte ${ }^{8}$. C'est le fondement de bien des méthodologies, explications de textes et autres, et le but ultime de nombre de lectures critiques, d'articles universitaires persuadés de maintenir à ce stade leur propos dans des procédures cognitives au plus près d'un discours de savoir et non de jugement. Or l'ouragan relativiste objecte que tout cela est une illusion et que la richesse informative comme la complexité structurelle sont des perceptions relatives à chaque sujet selon ses compétences ${ }^{9}$. Un spécialiste d'Aragon pourra trouver que Le Paysan de Paris est beaucoup plus riche qu'Aurélien alors qu'un lecteur ordinaire percevra dans le second roman un autre mode de complexité ou de richesse (métaphorique, historique) que la difficulté du texte surréaliste laissait pour lui lettre morte. D'autre part, la richesse et la complexité sont à concevoir comme des valeurs idéologiquement motivées qu'une communauté à un moment donné a élues comme critères de jugement de l'œuvre littéraire, alors que d'autres auront privilégié l'inspiration ou l'authenticité, - ces valeurs peuvent d'ailleurs coexister comme en témoigne la valorisation du haiku ou du fragment par exemple. Il ne s'agit pas de soutenir l'inanité des critères objectifs de l'évaluation, ce qui a déjà largement été fait par la théorie esthétique des dernières années du $\mathrm{XX}^{\mathrm{e}}$ siècle, mais de montrer les difficultés de nos pratiques dès lors que la pensée de la valeur s'y introduit. Car la complexité ou la richesse ont ceci de différent : si plus qu'aucune autre elles légitiment nos pratiques d'enseignement, c'est sans doute que ces valeurs fondées sur l'enplus apparaissent, pour cette raison, comme mesurables et quantifiables ou objectivables sous forme d'inventaire dénombrable. En somme richesse et complexité sont symptomatiques de la manière dont la valeur disparaît sous les masques du savoir et plus généralement du traitement de la valeur en régime de savoir. Un texte exigeant en termes de connaissance (requises ou possibles) - c'est-à-dire en tant qu'objet du savoir - serait forcément riche en valeur littéraire. On pourrait ajouter que la valeur de vérité ou d'objectivité des telles qualités semble également validée par le caractère inépuisable des commentaires sur l'œuvre, qui d'une certaine manière, fonde la littérature comme discipline universitaire. On ne peut ignorer la

8. Voir la critique serrée que propose Gérard Genette (L'Oeuvre de l'art, t. II, La relation esthétique, Seuil, 1997) des trois valeurs que sont unité, complexité et intensité telles que les défend par exemple, à la fin des années 50, l'esthétique analytique de Monroe Beardsley, valeurs que Genette relie aux conditions de la Beauté selon Thomas d'Aquin. (p. 94)

9. «It should be noted that « structural complexity » and information-richness » are of course subject-relative as « qualities » and also experentially subject-variable » (B. Herrnstein-Smith, op. cit., p. 52) 
circularité de l'argument : le renouvellement permanent des lectures de l'œuvre prouve qu'il s'agit d'une grande œuvre c'est-à-dire d'une œuvre riche, mais en retour, c'est la conception de l'œuvre comme détentrice d'un infini de richesses en attente d'être découvertes qui fonde la légitimité et « l'objectivité » de toute nouvelle interprétation. En réalité, le commentaire réussi est en lui-même producteur de richesse et de complexité, ce qui ne lui ôte aucunement sa légitimité et suffit à lui attribuer une valeur supérieure dans la hiérarchie des commentaires sans avoir besoin d'invoquer le critère d'objectivité de l'interprétation.

Le développement de l'enseignement de la littérature contemporaine à l'université fournit également un bon exemple des apories de la valeur, car le chercheur et l'enseignant se trouvent dans une position à peu près inédite et méthodologiquement difficile. Il s'agit d'une part pour les spécialistes de déterminer le territoire de la recherche critique sur le contemporain (et de le défendre) par opposition aux pratiques journalistiques, en minorant l'importance du geste critique à proprement parler au profit du geste théorique : " Il ne s'agit pas seulement de signaler ni d'évaluer une œuvre [travail de la critique journalistique] mais de montrer comment elle s'insère dans un ensemble de questions qui lui sont - ou non - contemporaines, comment elle est conçue, par quel travail d'écriture sont obtenus les effets auxquels elle prétend etc. ${ }^{10} »$, tout en reconnaissant d'autre part que le « geste critique », lorsqu'il porte sur le contemporain, « consiste en l'obligation de justifier l'élection de tel ou tel texte, de tel ou tel auteur » et que « la question de la valeur est donc d'emblée pertinente $[. . .]^{11} \gg$. En effet, dans la mesure où le système fonctionne qu'on le veuille ou non jusqu'à nouvel ordre comme une instance de valorisation, inscrire Michel Houellebecq ou Jean Echenoz au programme d'un cours, c'est déjà destiner ces auteurs à devenir comme Balzac des valeurs-étalons par rapport auxquelles se détermineront les valeurs des Lettres. Il est en effet certain que dès lors qu'on engage des ressources universitaires (l'organisation d'un colloque par exemple) autour d'un écrivain, on est entraîné irrésistiblement dans une logique de justification scientifique de la valeur de cet auteur. Le paradoxe universitaire est le suivant : tout sacrifice à la positivité (à l'illusion de positivité) s'accompagne d'une consécration, en vertu du lien naturel entre savoir et pouvoir. S'il n'y a pas de moyen véritablement scientifique pour légitimer la place de Pierre Michon, Richard Millet ou Pascal Quignard au sommet de la hiérarchie universitaire, le champ du contemporain engage à justifier les présupposés qui dirigent ces choix. La pratique du « contemporain » à l'université est le plus souvent l'occasion d'une pratique consciente et maîtrisée de la valeur et d'un regard critique sur les « grands récits » de l'histoire littéraire, mais elle est exemplairement soumise aux

10. Dominique Viart, «De la littérature contemporaine à l'université : une question critique » dans L'Atelier de Fabula, http ://www.fabula.org/atelier.php

11. Dominique Rabaté, « Au risque du contemporain », La Valeur, dir. D. Vaugeois, Revue des Sciences Humaines, ${ }^{\circ} 283$, juillet-septembre 2006. 
risques inhérents à la parole en milieu institutionnel et en régime de savoir.

C'est ainsi par exemple que Dominique Rabaté a pu proposer de définir l'œuvre majeure comme œuvre « symptomatique des questions et des réponses qu'un moment de l'art appelle (ou qu'un auteur invente en prenant son époque à contrepied) ${ }^{12}{ }$. Or, comme D. Rabaté lui-même le suggère, évaluer le contemporain en termes de symptôme ne permet pas de résoudre le problème de la valeur. Car tout d'abord la notion de symptôme est bien entendu une valeur historiquement déterminée : établir un livre comme œuvre majeure parce qu'il est symptomatique, c'est poser comme valeur implicite la place de l'œuvre dans l'actualité de son temps (qu'elle l'emblématise ou en montre les limites), ce qui n'a rien d'une vérité absolue mais participe des valeurs de la modernité, que l'on peut mettre en perspective avec d'autres époques où la littérature cherchait au contraire à viser par exemple l'éternel ou l'universel. D'autre part, le symptôme comme valeur pourrait être aussi la conséquence des différentes théories descriptives de l'histoire littéraire : nous avons constaté que les œuvres du passé dont la valeur est reconnue ont été en effet symptomatiques de leur temps; cette pratique descriptive devient subtilement prescriptive dès lors qu'elle appuie la sélection et la justification des œuvres du présent. Cette relativité mise à part, l'idée de symptôme pose un problème supplémentaire. Comment juger de l'importance historique d'une œuvre à partir de son présent ? Ainsi la valeur des œuvres de Michel Houellebecq tient à l'idée que l'on se fait de ce que doivent être les symptômes de notre époque. Tout ceci n'est guère neuf et ne fait qu'illustrer la maxime selon laquelle on ne peut être juge et partie à la fois. « Le contemporain », qui, en France plus qu' ailleurs, constitue un domaine à part avec ses colloques et ses spécialistes et qui représente en quelque sorte, dans une université française moins concernée que ses homologues outre-antlantique par la remise en cause des œuvres classiques, un des lieux de mise à distance du canon, constitue une intéressante illustration des difficultés de la valeur.

Le second présupposé mis à mal par la pensée relativiste est celui de l'éducation critique. C'est le plus pervers parce qu'il constitue non seulement un argument de légitimation des textes choisis - par une autorité cultivée, celle de l'enseignant mais de la pratique universitaire elle-même qui repose sur l'idée de compétences acquises et à acquérir en vue d'une progression. Or l'idée d'un apprentissage de la lecture des textes est implicitement reliée à celle de la reconnaissance de la valeur d'un texte, d'une meilleure appréciation des œuvres dans leurs différences qualitatives et donc à l'idée que la valeur littéraire des textes proposés est une propriété déductible de l'analyse, ce qui bien sûr nous replace, comme avec l'exemple précédent, dans une démarche positiviste où la valeur serait une caractéristique de l'objet, où encore une fois les logiques de savoir déterminent les processus d'éva-

12. D. Rabaté, « La Valeur en question », Thélème (Revista Complutense de estudios franceses), $\mathrm{n}^{\circ}$ 15, Université Complutense, Madrid, 2000. 
luation. C'est à cela que n'échappe pas Gaëtan Picon malgré son souci de prendre en compte en 1953 une certaine relativité lorsqu'il écrit dans L'Ecrivain et son ombre :

Parmi tant d'admirateurs lucides de Baudelaire et Mallarmé, il n'en est pas deux qui vivent identiquement l'admiration commune, pas deux qui la situent au même niveau. De l'individu qui l'éprouve, l'expérience esthétique retient beaucoup : et notre hiérarchie des valeurs est toujours quelque peu personnelle. Mais l'esthétique n'exige nullement l'identité des réactions ou une échelle de valeurs uniques. Elle requiert seulement l'objectivité des valeurs. Nous ne lisons pas Racine comme le lisaient Louis Racine, Voltaire ou Sainte-Beuve : mais son œuvre est lourde de beautés réelles, et appelle un sentiment qui lui rende justice $^{13}$.

Il s'agit encore une fois d'affronter la question de la subjectivité ou de l'objectivité des valeurs et de l'évaluation. Le tour de force argumentatif auquel veut se livrer Picon ici consiste à reconnaître que les valeurs que l'on accorde à l'œuvre sont subjectives, variables, diverses à condition d'admettre en même temps qu'elle se fondent néanmoins en tant que réactions dans la substance d'un objet, l'œuvre d'art. Les valeurs que nous accordons à l'œuvre sont la manifestation de sa valeur fondée dans une série de qualités. Il conclut ainsi peu ou prou son ouvrage sur cette définition de la formation de ce qu'il appelle une « sensibilité compétente » : « sa maturation n'est rien d'autre que le progrès d'une lucidité liée à l'enrichissement de l'expérience » (269). Or cette lucidité une fois acquise aura pour tâche de « distinguer autrement que par une réaction sentimentale qui doute d'elle-même les fausses et les vraies clartés » (269). Cette clairvoyance, cette perspicacité du lecteur cultivé telle que la pense Picon, suppose alors qu'il y ait dans l'œuvre un secret à percer, une vraie clarté, que l'œuvre soit porteuse dans sa structure même de valeurs que l'on apprend à connaître (dont on peut avoir une connaissance positive) et qui la valident comme œuvre d'art ${ }^{14}$.

Le dispositif des Lettres soumis aux exigences contradictoires de l'évaluation et du savoir nous contraint à l'exercice contradictoire d'un relativisme théorique qui met en bonne et due place la pensée moderne des valeurs, et d'un essentialisme pratique ${ }^{15}$ qui, par définition, évacue les valeurs telles que la pensée relativiste moderne les définit. La valeur ne peut être ni éliminée ni codifiée sous peine de se défigurer en faux savoir.

13. Gaëtan Picon, L'Écrivain et son ombre, (1953), « Tel», Gallimard, p. 249. Je souligne.

14. Pour cette raison, la définition de l'esthétique comme « méthodologie du jugement » qu'il propose n'est guère convaincante.

15. Nous serions inévitablement victimes des « illusions objectivantes » que Genette pose comme constitutives de toute pratique évaluative (La Relation esthétique, op. cit.). 
Enfin, l'enseignement des lettres participe de ce que l'on conçoit comme la démocratisation d'une culture d'élite et permet d'offrir à l'ensemble de la société une part de ce « capital symbolique » qu'offre la littérature. Or un tel discours social mérite d'être interrogé. En effet, il implique que l'accès à la culture soit considéré comme étant de fait une expérience supérieure aux autres, fournissant l'accès à un « mieux vivre » par comparaison avec l'expérience du milieu familial des classes moyennes, celui de la culture télévisuelle et des bestsellers. La discipline reste en effet pour une grande part dépendante du modèle de la société humaniste fondée sur la culture lettrée et qui a constitué en Europe le ciment de la nation moderne par le biais de l'école républicaine. La démystification de ce que B. Herrstein Smith considère comme une croyance tout aussi relative que les autres, a alors le mérite de nous forcer à faire la part, dans la valorisation de l'expérience littéraire, de ce qui relève d'une idéologie héritière de modèles politiques. L'ambiguïté de la leçon du film L'Esquive d'Abdellatif Kechiche tient à ce que les vertus de l'expérience que constitue la lecture de Marivaux sont toujours récupérables en termes de l'accession de jeunes exclus futurs délinquants au statut de bons citoyens.

La pensée moderne de la valeur, à la différence de la pensée ancienne, établit le concept de valeur, ou son pluriel, comme le vis-à vis critique du concept d'essence. Nous vivons dans un monde de valeurs relatives et non d'essences absolues. Mais le « couteau de la valeur », selon la formule de Barthes, est à double tranchant : outil épistémolgique au service de la dénonciation des illusions, celle du savoir absolu et des vérités éternelles, la valeur est aussi, nécessairement, ce qui n'est que valeur (et non savoir) et donc par nature, depuis que la généalogie nietzschéenne a ouvert la modernité par la question de la valeur de la valeur ${ }^{16}$, hautement soupçonnable. La Valeur, c'est alors finalement rien moins que la « Terreur dans les Lettres » avec la double face que lui reconnaît Jean Paulhan. D'un côté la valeur participe de la terreur ancienne, celle des prisons dogmatiques de l'universel et de l'en soi, de l'autre, souvent mise au pluriel, concept clé d'un relativisme moderne, elle apporte avec elle une violence révolutionnaire qui fait sauter les barrières à l'abri desquelles se développe le savoir disciplinaire.

\section{Les vases communicants : la littérature en milieu fluide}

L'issue serait donc d'accorder une pleine place à la valeur dans les discours, de parler du point de vue de la valeur. Cependant, accueillir dans son discours la dimension de la valeur, c'est ouvrir ce dernier à des niveaux d'analyse divers et complexes à articuler. L'attention accrue prêtée à la dimension axiologique des œuvres par la réflexion récente sur la littérature a fourni l'exemple d'une extension

16. Nietzsche, La Généalogie de la morale, Avant-propos, paragr. 3, 6, traduction de C. Heim, Gallimard. 
du champ d'étude à ce que les influences formalistes avait pu amener à considérer comme un plan extérieur à l'analyse du « texte » lui-même, qu'il s'agisse de la sociosémiologie des contenus qui étudie l'inscription de l'idéologique dans les structures formelles du texte ${ }^{17}$ ou de la revalorisation des problématiques de l'intentionnalité qui repense, du point de vue moral, la relation entre les choix existentiels d'un individu et l'acte d'écriture ${ }^{18}$. Mais la prise en compte des mécanismes de l'évaluation au sein de nos discours oblige à une ouverture plus radicale des délimitations que les méthodes du savoir disciplinaire imposent à leurs objets.

On a l'habitude d'avancer la nécessité d'une distinction entre, d'une part, au singulier d'abord (en un sens quantitatif marqué sur une échelle par le plus ou le moins) valeur de la littérature et valeur des œuvres, et, d'autre part, au pluriel, les valeurs littéraires (celles dont nous parlions plus haut : complexité, symptômaticité etc...) et les valeurs sociales (politiques, culturelles, économiques, morales.). Or cette distinction conceptuelle et artificielle brouille la réalité plus qu'elle ne la clarifie. Car le véritable enjeu de la (ou des) valeur(s) tient précisément aux incessants passages de l'un à l' autre de ces niveaux. Pour reprendre le paradigme économique, J.-J. Goux suggère que nous ne devrions pas parler de valeur de la littérature (une entité autonome, à laquelle on attacherait une valeur) mais de valeur-littérature ${ }^{19}$ (une entité qui n'existe que par son évaluation). Or la formule « valeur-littérature » est une invitation à penser le lien entre la valeur sociale de la littérature (sa valeur au sein de la société à côté d'autres formes d'arts mais aussi d'autres activités ou fonctions) et la valeur d'une œuvre à l'intérieur de la littérature. Il est difficile de séparer la question de la valeur de la littérature et de la valeur en littérature parce qu'évaluer une œuvre c'est faire appel à une définition de la littérature et inversement, la valeur de la littérature dépend de celle accordée aux œuvres. Mais cette valeur accordée aux œuvres dépend elle-même de la valeur de la littérature dans la société et des valeurs qui y circulent. De la même manière, la réflexion sur le contenu axiologique de l'œuvre, c'est-à-dire les valeurs que l'oeuvre met en jeu - la démocratie chez Hugo ou chez Tocqueville par exemple -, n'est donc pas à concevoir sur un plan radicalement différent de celle sur la valeur du littéraire. On constate alors en premier lieu que les catégorisations axiologiques perdent de leur distinction, ensuite que c'est bien en réfléchissant en termes de valeur qu'on a le plus de chance de comprendre les relations entre l'art et la société, enfin que la valeur en littérature participe d'un grand jeu qui dépasse les frontières du littéraire. Wayne Booth après Bourdieu a bien montré que nous manions sans cesse des valeurs éthiques tout en croyant rester dans les limites de l'esthétique. L'une

17. Vincent Jouve, Poétique des valeurs, PUF, 2001.

18. Voir à ce sujet les analyses fines de Michel Jarrety sur Camus, Char et Cioran dans La Morale dans l'écriture, PUF, 1999.

19. Sur le modèle de la valeur-esprit de Valéry « La Liberté de l'esprit », (1939) Regards sur le monde actuel, 1945). 
des valeurs esthétiques essentielles de la critique post moderne à laquelle nous appartenons à savoir celle de l'œuvre ouverte fondée sur la puissance d'interrogation de l'œuvre est une valeur éthique.

Ne pas prendre en compte ce circuit de la valeur, c'est risquer de fausser l'analyse. Arrêtée dans son mouvement par les limites d'une méthodologie, la valeur se défigure et retrouve le masque grimaçant de l'hydre essentialiste. C'est d'une certaine manière le péril que frôle le récent livre de William Marx. En effet L'Adieu à la littérature ${ }^{20}$ est une enquête sur la dévalorisation de la littérature depuis le $\mathrm{XVIII}^{\mathrm{e}}$ siècle. Et il propose d'expliquer cette réalité socio-historique par les seuls déterminismes internes à la littérature : « ce qui est ici tenté, c'est moins une histoire des relations de la littérature et de la société qu'une étude de la façon dont la littérature elle-même réfléchit son propre rapport au monde » (14). La « baisse d'influence » de la littérature et la « crise existentielle » où elle se trouve seraient à comprendre en relation avec « la littérature de l'adieu à la littérature » symboliquement représentée par Rimbaud mais préparée tout au long du XIX ${ }^{\mathrm{e}}$ siècle par « l'enfermement dans la forme ». La littérature serait responsable, par l'image qu'elle donne d'elle-même (autodépréciation du langage, refus du monde, etc), du rétrécissement supposé de sa sphère de pouvoir. Si l'hypothèse est séduisante et assez caractéristique du retour critique des praticiens de la littérature sur leur objet en une sorte de mea culpa par procuration qui excuse la désertion des lecteurs, elle n'est guère convaincante ${ }^{21}$ car il manque un jalon important. En effet, la valeur qui est donnée à la littérature, à l'intérieur de la littérature, ne peut se traduire immédiatement en réalité sociale. Le passage entre les domaines constitue l'impensé de la théorie. Les valeurs que la littérature promeut à l'intérieur du champ sont nécessairement soumises à l'influence des valeurs extérieures au champ ${ }^{22}$ et en conséquence - c'est la logique des vases communicants - , cette dévalorisation interne ne peut exister dans le champ social qu'à condition d'entrer en résonance négativement ou positivement avec les valeurs de cette société. Le procès fait au « formalisme » par l'auteur en témoigne au premier chef. Lorsque W. Marx écrit à propos de cette littérature « formaliste » (Valéry ? L'Oulipo ? Celan ? l'ensemble reste indéterminé) :

quand la langage perd sa transparence au réel, à la limite la littérature n'est plus censée parler de rien, elle fonctionne comme un objet autonome fermé sur soi ; et ne parlant plus de rien, elle est elle-même

\footnotetext{
20. op. cit.

21. Pour plus de détails, voir le compte-rendu critique que je consacre au livre : « Qui a tué la littérature? », Acta Fabula, printemps 2006, (volume 7, numéro 1), http ://www.fabula.org/revue/document1154.php.

22. L'autonomie bourdieusienne ne signifie par imperméabilité : l'autonomie du champ ne peut se proclamer, par le biais des ?uvres et des auteurs, qu'en un langage commun.
} 
destinée à n'être plus rien - ou plus qu'un objet pur destiné à une consommation de plaisir, détaché de tout rapport avec les réalités du monde (160),

on est droit de se demander si l'absence d'analyse permettant d'accorder un sens littéraire et linguistique précis à des expressions telles que « transparence au réel » ou « objet autonome fermé sur soi » ne se justifie pas précisément par les valeurs à partir desquelles s'énoncent de tels jugements. En somme, il ne s'agit pas là de la description du fonctionnement littéraire des œuvres comme échec d'une forme-sens, mais d'un jugement, tributaire d'une critique des valeurs élitistes d' « autonomie » et conjointement d'une promotion des valeurs de « transparence » telles qu'elle se déclinent (sincérité, témoignage...) et s'illustrent dans la société aujourd'hui et telles qu'effectivement elles programment la dévalorisation d'une littérature qui ne leur correspond pas. La question de la relation de la littérature et de la société est donc à la fois ce qui motive l'essai et la grande absente de l'équation. La déclaration de disgrâce de la littérature ne peut avoir de légitimité en l'absence d'une interrogation sur les structures culturelles et sociales plus générales, notamment la crise des humanismes littéraires nationaux après 1945, dans lesquelles cette disgrâce est censée se produire.

\section{Discours de savoir et discours de valeur}

En somme, le tournant pris par une histoire littéraire qui problématise la valeur dans le dernier quart du $\mathrm{XX}^{\mathrm{e}}$ siècle est à la fois le signe positif d'une volonté de sortir des représentations insulaires de la littérature et l'exemple de la difficulté que rencontre une théorisation proprement littéraire de la valeur. Le livre de W. Marx témoigne du difficile pari d'une sociologie de la littérature sans sociologie et révèle plus généralement la situation ambiguë des études littéraires actuelles qui ont pris acte des ouvertures formidables de la théorie bourdieusienne mais ne lui pardonnent pas tout à fait d'avoir dissous, dans le même temps qu'elle offrait de penser la littérature en champ autonome, la « littérarité » des questions littéraires. En effet assumer pleinement le point de vue la valeur, ne serait-ce pas être contraint d'accepter, selon la célèbre formule, que la littérature soit soluble dans la sociologie ? Car, si précieuse et bénéfique qu'elle soit pour la réflexion sur la littérature, la conscience sociologique, telle qu'elle s'est formée à partir de Bourdieu, conduit irrésistiblement à ce relativisme cynique dont nous parlions plus haut, qui disqualifie la pertinence de toute évaluation des œuvres en termes de qualité littéraire. Selon Jean-Jacques Lecercle nous avons en effet le choix entre deux attitudes, l'approche relativiste d'une part : « Il n'y a pas d'excellence ou de génie en littérature. Il n'y a plus de canon. Tous les textes se valent » et le « cynisme »d'inspiration sociolo- 
gique d'autre part : " Pour elle non plus il n'y a pas de grands textes, ou plutôt leur grandeur est sociologiquement déterminée ${ }^{23} \gg$ Or, ces deux attitudes ne constituent pas un choix mais bel et bien deux stades d'une même approche qui conteste en définitive l'existence de la valeur littéraire : le premier en récusant la validité du point de vue hiérachique de la valeur (il n'y a pas de valeur littéraire), le second en donnant de la canonisation historique de telle ou telle œuvre « une explication indépendante de [sa] qualité littéraire » (il n'y a pas de valeur littéraire). Ceci joint aux récentes contestations dans le champ de l'esthétique des fondements rationnels des discours évaluatifs soutenus par exemple par Jean-Marie Schaeffer et Gérard Genette $^{24}$ aboutit à placer le discours littéraire dans une situation intenable : toute pratique littéraire est une évaluation mais aucune évaluation n'est littérairement légitime.

Pourtant cette aporie ne tient qu'à l'intérieur d'une pensée qui reste soumise à un régime de connaissance particulier. L'invalidation de la valeur se fait en relation au modèle du discours de savoir. Or ce combat du savoir et de la valeur est d'abord dû au cadre culturel et historique à l'intérieur duquel nous nous trouvons. Il tient à la place institutionnelle ambiguë de la discipline littéraire, à la difficulté de justifier aujourd'hui, politiquement et socialement, son enseignement ainsi que les ressources consacrées à sa recherche, si la discipline elle-même n'est pas « dans le savoir »? Plus globalement, ce soupçon porté sur la valeur au nom des logiques de savoir relève, je le mentionnais plus haut, de cette disparition d'un humanisme qui entre 1789 et 1945 en Occident a fondé les nations modernes sur les vertus des Lettres comme ciment communautaire comme l'a brillamment montré Peter Sloterdijk ${ }^{25}$. L'enseignement des Lettres aujourd'hui dépossédé de cette légitimité semble ne pouvoir se justifier qu'en s'alignant sur les discours de savoir. Mais ce cadre historique se double de (ou trouve écho dans) le cadre philosophique, à la fois épistémologique et éthique, à l'intérieur duquel les théories de la valeur pensent cette dernière. Même chez Bourdieu, pensée comme instrument de stratégies de domination par lesquelles un capital symbolique est converti en capital matériel, la valeur est l'objet d'une démystification ${ }^{26}$ et son discrédit en tant qu'illusion tient bien à son statut de "faux-savoir ». Même chose chez Genette, où les prédicats esthétiques apparaissent comme des masques trompeurs et l'évaluation comme une pratique schizophrène inquiétante. Tout se passe alors comme si l'acharnement critique de la pensée relativiste de la valeur, qu'il s'agisse d'un relativisme sociologique ou du relativisme subjectiviste des philosophes de l'esthétique, alors

23. Jean-Jacques Lecercle, « Y a-t-il un progrès en littérature? », Vox Poetica, 2003, http ://www.vox-poetica.com/t/progres.htm

24. Voir, pour une approche critique plus détaillée de ces questions, D. Vaugeois, « Faut-il avoir peur des valeurs? » dans La Valeur, op. cit.

25. Règles pour le parc humain (1999), trad. Olivier Mannoni, Mille et une Nuits, 2000.

26. C'est ce qu'évitent, par exemple, les travaux de N. Heinich. 
même qu'il prend acte de l'empire des valeurs et démontre brillamment les illusions du discours purement factuel sur l'œuvre d'art, manifestait le regret inavoué d'un impossible savoir de la valeur. Le relativisme serait un positivisme militant en même temps peut-être qu'un idéalisme déçu. Le discours de valeur, dans une telle conception n'est alors pas seulement mis de côté comme douteux et illégitime mais est tout simplement impossible à penser. Dans la perspective relativiste subjectiviste de la valeur qui est celle de Genette, la valeur n'a pas de place ; la notion même disparaît. Car la valeur est précisément ce qui n'est ni personnel ni universel : le champ d'application des valeurs dépassant par définition le plan strictement individuel et impliquant la mise en relation conçue comme comparaison et mise en commun. Or ce n'est qu'en reconnaissant la spécificité du discours de valeur face au discours de savoir qu'il devient possible à la pensée littéraire d'éviter le cynisme tout en conservant la lucidité qu' apportent à nos pratiques évaluatives la conscience sociologique et l'anti-essentialisme philosophique.

Donner sa pleine place à la valeur dans les lettres consisterait alors à redéfinir la place de la subjectivité dans les discours, subjectivité sommée de s'effacer devant l'objet dans les logiques du savoir. Le discours universitaire et critique doit pour reprendre la célèbre définition de Baudelaire accepter d'être « partial [...], passionné [...], politique, c'est-à-dire fait ...] à un point de vue exclusif mais qui ouvre le plus d'horizons. ${ }^{27}$ ». La dimension « politique » de la tâche universitaire peut aller jusqu'à se formuler en termes de lutte idéologique, par où se pense, selon Jean-Jacques Lecercle, la relation au canon (défini à la fois dans son historicité et sa nécessité ${ }^{28}$ ) comme défense et attaque, comme protection et révision. La notion de responsabilité mérite d'être repensée en relation avec celle d'engagement qui, loin d'être inconcevable au sein de la critique scientifique ou de la relation pédagogique, doit au contraire s'y réinviter.

27. Baudelaire, « Salon de $1846 »$, Critique d'art suivi de critique musicale, Gallimard, 1992, coll. « Folio », p. 78.

28. J.-J. Lecercle, « Y a-t-il un progrès en littérature? », art. cit. 